\title{
MKL-1 regulates the stem cell marker. CD44 in breast cancer cells
}

\author{
Zhou-Tong Dai ${ }^{\text {1a }}$, AoYao ${ }^{1 a}$, Yuan Xiang ${ }^{1 a}$, Jia Peng Li ${ }^{1}$, Wei Guo ${ }^{3}$, Hui-Min Zhang ${ }^{1}$, Feng Huang ${ }^{1}$, Han-Han Li ${ }^{1}, Z^{-}-J_{i a n g}$ \\ Zhang ${ }^{1}$, Hui $\mathrm{Li}^{1}$, Xing Hua Liao ${ }^{1,3 a^{*}}$ \\ ${ }^{1}$ Institute of Biology and Medicine, Wuhan University of Science and Technology, P.R.China, 430000 \\ ${ }^{2}$ Key Laboratory of Industrial Fermentation Microbiology, Ministry of Education and Tianjin, College of Biotechnology, Tianjin \\ University of Science and Technology, P.R.China, 300457 \\ ${ }^{3}$ Shenzhen Ritzcon Biological Technology Co., LTD, Shenzhen, Guangdong, P.R.China, 518000
}

\begin{abstract}
CD44, cluster of differentiation 44 is a typical marker of stem cells. At present, it has been found that CD44 is prevalent in various human malignant tumors, but its expression regulation mechanism is still not clear. The initiation of gene expression, the modification of RNA levels, and the regulation of protein levels are the main factors affecting the expression level of genes, and the most critical one is the regulation of gene expression by signaling pathways. Up to now, there has been no report on the role of MKL-1 in the cloning of the cd44 promoter. Therefore, this study intends to clone the cd44 gene promoter, construct its luciferase reporter gene vector, transfect the MKL-1 overexpression vector, and analyze how it affects transcriptional activity, in order to further study the expression regulation of cd44. The mechanism provides a powerful tool in the future.
\end{abstract}

\section{Introduction}

CD44, cluster of differentiation 44 is a typical stem cell marker. Initially in the study of breast cancer, CD44 was first discovered and confirmed to be a solid tumor stem cell surface marker molecule [1]. Subsequently, it was confirmed that cancer stem cells in solid tumors such as brain cancer, pancreatic cancer, melanoma, colon cancer, and liver cancer all have CD44 expression [2-7]. CD44 is a type I transmembrane glycoprotein that functions as a cell membrane receptor involved in a variety of biological processes in cells, such as survival, proliferation, and differentiation [8-10]. For example, CD44 can act as a receptor for hyaluronan signaling into intracellular activation of the downstream signaling pathway Wnt/ $\beta$-catenin. CD44 is a unique adhesion molecule that mediates the migration of tumor cells and adheres to the matrix in the cellular microenvironment, thereby strengthening the invasion ability of tumor cells and promoting the proliferation of tumors. Interference experiments show that inhibiting the expression of CD44 can significantly reduce the ability of tumor cells to form clones and form tumors [11]. Gastric adenocarcinoma highly expresses CD44, and multiple human gastric cancer cell lines (NCI-N87, AGS, MKN-28, MKN-45, MKN-74), it was found that CD44 is expressed in gastric cancer cells and used as a surface of gastric cancer stem cells Marker [12]. Some research has found that CD44+ and CD24+ cells were isolated from cancer tissues of 5 patients with gastric cancer. CD44+/CD24+ can be used as markers for gastric cancer stem cells by comparing the self-renewal, differentiation and tumorigenicity of different phenotype tumor cells [13].

MKL-1 (MRTF-A, Myocardinrelated transcriptional factor) is a transcriptional regulator originally discovered as a transcriptional cofactor for the Serum response factor (SRF). It is involved in regulating the expression of smooth muscle cell-specific genes [14; 15]. Serum response factor (SRF) is a transcription factor. Some immediate early genes were regulated by SRF [16]. Although MKL-1 does not activate SRF in a resting state of cells, MKL activates SRF after a series of genes [17]. In Prostate Cancer Cells, some research identified that one compound could block MKL transcription to inhibit prostate cancer cell invasion [18]. In HCC some researches demonstrated that depletion of MKL-1 curbs HCC xenograft growth [19], but there is no study related to MKL-1 and cd44.

In this study, a plasmid vector with a luciferase reporter gene for the CD44 promoter was successfully constructed. The value of luciferase was then detected after transfection of the overexpression vector of human MKL-1 in the HEK293T cell line and SGC-7901 cell line. The results showed that the transcriptional activity of CD44 was significantly enhanced after transfection of MKL-1 overexpression vector.

And further determine the site of action of mkl-1 and CD44 by combining site-directed mutagenesis.

This study demonstrates that MKL-1 can promote the promoter activity of CD44 and lay the foundation for subsequent research.

\footnotetext{
*Corresponding author: xinghualiao@hotmail.com
} 


\section{Methods}

\subsection{Cell culture and CD44 promoter plasmid construction}

Human gastric cancer cell line (SGC-7901) was obtained from American Type Culture Collection. SGC-7901 cells were grown in Roswell Park Memorial Institute 1640 Medium (contained with $10 \%$ fetal bovine serum and $1 \%$ pen strep) at 37 with $5 \% \mathrm{CO}_{2}$ in incubator. And Human Embryonic Kidney 293T (HEK293T) cells line was obtained from American Type Culture Collection. HEK293T cells were grown in Dulbecco's modified Eagle's medium (DMEM) (contained with 10\% fetal bovine serum and $1 \%$ pen strep) at 37 with $5 \% \mathrm{CO}_{2}$ in incubator.primer synthesis and target gene sequencing were performed by company Sangon Biotech (Shanghai) Co., Ltd.

The CD44 promoter reporter plasmid was constructed by inserting the core fragment of the CD44 promoter into the PGL-3 promoter plasmid. The Xhol locus is selected as the fragment insertion site. Using the genome of human HEK293T cells as a template, a core fragment of the CD44 promoter (from -1966 to +33 ) was obtained by polymerase chain reaction, and Xhol restriction sites and homologously recombined fragments were introduced at both ends of the primers. The designed primers

Sense primer:

5' CGgTACCAGCCCATTTTATACTTGAGCA 3' Anti-Sense primer:

\section{5' GCGCTAGCCCACCAAAACTTGTCC 3'}

PCR conditions were: $95^{\circ} \mathrm{C}$ for $10 \mathrm{~min} ; 95^{\circ} \mathrm{C}$ for 1 $\min , 55^{\circ} \mathrm{C}$ for $30 \mathrm{~s}, 72^{\circ} \mathrm{C}$ for $2 \mathrm{~min}, 36$ cycles; $72^{\circ} \mathrm{C}$ for $10 \mathrm{~min}$; reaction was terminated at $4^{\circ} \mathrm{C}$.

After the reaction is over, the corresponding PCR product is taken for gel electrophoresis.

The target fragment with the sticky ends and the PGL-3 plasmid fragment were obtained by restriction enzyme restriction of $\mathrm{kpn} 1$ and nhe1, and the target fragment was inserted into the PGL-3 vector using a ligase to obtain the target vector. The amount required for the ligation was calculated and the vector and the target fragment were added to the ligase system in a proportioned manner, and the ligation was performed at $16^{\circ} \mathrm{C}$. for $24 \mathrm{~h}$. The ligated product was transferred into the competent state, and an experimental group and a control group with ampicillin-free colonies were set up. Monoclonal colonies were picked from the test group and cultured in LB and 180r/min shaker culture. Plasmids were extracted using a small extracted plasmid kit (Solarbio) and sequenced.

\subsection{Luciferase reporter assays}

Selected HEK293T cells and SGC-7901 cells that were grown in logarithmic stages were prepared with a single cell suspension after trypsin digestion. Cells were counted and seeded into 6 -well plates at a density of $1 \mathrm{x}$ $10^{5}$ cells/well. After cells grow to $50 \%$, use PEI transfection reagent, follow the instructions to perform plasmid transfection. After 6 hours of transfection, replace the fresh medium. After 48 hours of transfection, measure luciferase activity by SynergyTM 4 (Bioteck).

\subsection{Construction of CD44 promoter luciferase reporter plasmid}

You are free to use colour illustrations for the online version of the proceedings but any print version will be printed in black and white unless special arrangements have been made with the conference organiser. Please check with the conference organiser whether or not this is the case. If the print version will be black and white only, you should check your figure captions carefully and remove any reference to colour in the illustration and text. In addition, some colour figures will degrade or suffer loss of information when converted to black and white, and this should be taken into account when preparing them.

\section{Result}

\subsection{Construction of CD44 promoter luciferase reporter plasmid}

The PCR product of the target fragment obtained by PCR using the genome of human HEK293T as a template showed that the size of the target fragment was approximately $2000 \mathrm{bp}$, and the gel recovery efficiency was $225 \mathrm{ng} / \mu \mathrm{L}$. The PCR product is shown in Figure 1.

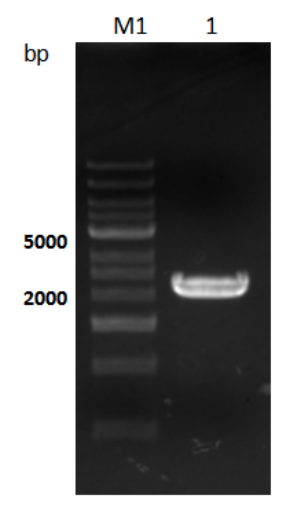

Fig. 1. Agarose gel electrophoretic analysis of PCR product.

The PCR products of PGL-3-Basic and the target fragment were double digested to obtain the same sticky ends Xho1. Then, the PCR product and the digested plasmid were separately purified, respectively, and then ligated overnight using T-4 ligase at $16^{\circ} \mathrm{C}$. Transform the ligation product into the SGC7901 and HEK293T cell lines. The result of the conversion of the ligation product was that the plasmid-free control group produced sterile colonies, the test group grew longer and the colony was larger, indicating that the activity of ampicillin was normal and the competent cells were available, and the grown colony was the colony of the connected product.

To confirm the recombinant plasmid, we used a restriction endonuclease XhoI to excise the recombinant plasmid and then verified by agarose gel electrophoresis. The results of the PGL-3 reassortment showed that after 
enzymatic digestion. As is shown in the Figure 2. The length of the recombinant plasmid was larger than the original plasmid of PGL-3, which was consistent with the design. Combined with the sequencing results, it was initially verified whether the plasmid was successfully constructed.

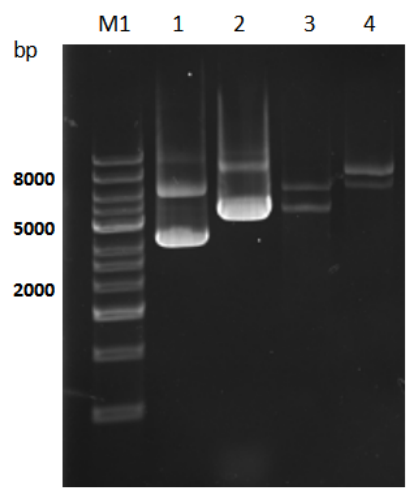

Fig. 2. PGL3-insulin luciferase recombinant plasmid digestion verification

)M1.DNA Market DL 10000. 1:PGL3-insulin luciferase plasmid. 2: PGL-3-insulin luciferase recombinant plasmid. 3: Digestion of PGL-3-insulin luciferase plasmid. 4: Digestion of PGL-3-insulin luciferase recombinant plasmid.)

To confirm the transfection efficiency of HEK293T cells, EGFP expression plasmids were used to transfect HEK293T cells. As shown in Figure 3, the transfection efficiency of the EGFP expression plasmid is approximately $70 \%$, indicating that the MKL-1 plasmid can also be transfected into HEK293T cells.

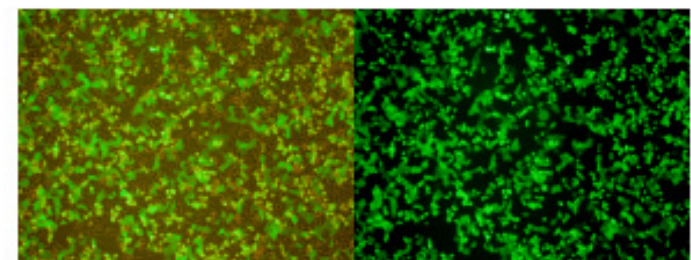

(1) Visible

(2) GFP

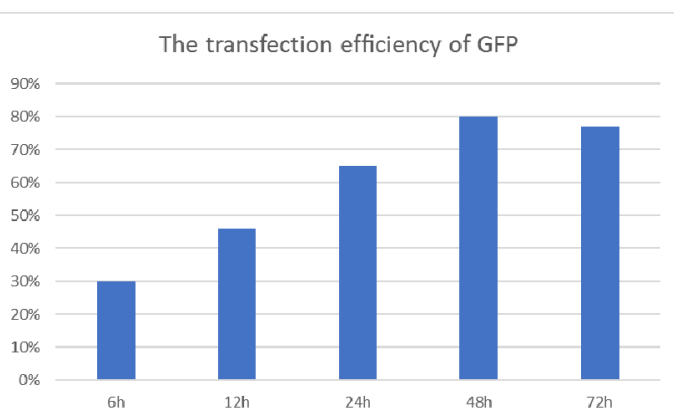

Fig. 3. The transfection efficiency of GFP .

((1) and (2) are the transfection of GFP after 48h.)

It can be shown in Figure 4 that overexpression of MKL-1 significantly increased the value of luciferase of cd44 compared to the control group transfected with pcdna3.1. And as shown in Figure 4. The same results were found in the SGC-7901 cell line.

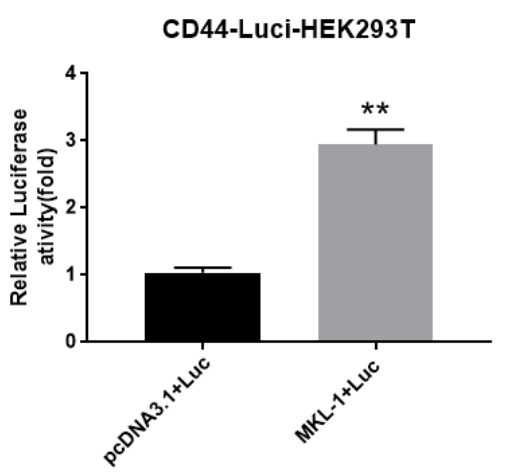

CD44-Luci-SGC7901

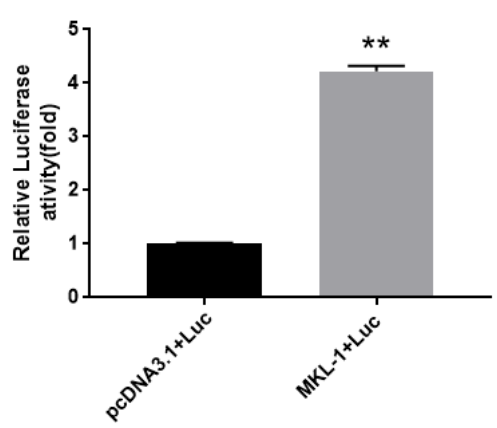

Fig. 4.MKL-1 can enhance the transcriptional activity of CD44. $(* * \mathrm{P}<0.01, * \mathrm{P}<0.05)$

\section{Conclusion}

Studies have shown that high CD44 expression is associated with the potential to activate gastrointestinal cancer cells metastasized in vivo. CD44v6 maximizes ligand-guided multi-directional behavior by disrupting CD44s interactions between cells and between cell-cell matrices. At the same time, CD44v6 acts as a hyaluronic acid receptor that binds to extracellular matrix and hyaluronic acid in the basal cell membrane, regulates cell movement and morphological changes, and CD44v6 positive cells are more easily associated with the high columnarity in the capillaries of the posterior vein. Endothelial cell binding makes it easier for tumor cells to enter the lymphatic system and circulatory system, while degrading hyaluronic acid, enabling the product to initiate angiogenesis, and enabling its own CD44v6 positive expressing cells to obtain stronger infiltration and metastasis [20-22]. These regulatory phenomena have initially formed the role of CD44 in tumor metastasis. We hypothesized that MKL-1 will affect cd44 and thus alter tumor migration.

MKL-1 is widely expressed in mammals and plays an important role in various physiological and pathological processes in the body. It has been reported that interference of MKL-1 expression in breast cancer cells can reduce the migration of tumor cells [23], and MKL-1 also plays a major role in tumorigenesis. The tumor-bearing ability of mice after MKL-1 knock-out of tumor cells was significantly reduced, and the ability to migrate to the lungs with blood circulation was also weakened [24]. MKL-1 regulates the activity of the Slug promoter by directly binding to the core region of the Slug promoter by interacting with Smad3, which is 
achieved through cis-acting elements of the GCCG-like motif [25]. There is an interaction between SCAI and MKL-1, and SCAI binds to RPEL and B-box of MKL-1 to inhibit its transcriptional activity without affecting the binding between MKL-1 / SRF [26]. The involvement of MKL-1 in tumor regulation is achieved in many ways. MKL-1, which is dependent on activation of Rho signaling pathway, can induce EMT by TGF- $\beta$ activation in MDCK cells [27]. All these evidences show that MKL-1 can be used as a new target for cancer treatment. Therefore, it is more important to understand how MKL1 plays a role in cells. However, it has not been reported that MKL-1 is involved in the regulation of the transcriptional activity of the cancer marker cd44.

This study investigated the effect of MKL-1 overexpression on the expression of cd44 promoter in lung cells at the cellular level. The luciferase reporter gene with the CD44 promoter core sequence was successfully constructed. The luciferase reporter assay showed that the expression of the cd44 promoter was significantly increased by up to 3 times after transfection of the MKL-1 overexpression vector, indicating that MKL-1 is essential for the transcriptional activation of the cd44 promoter. RTPCR experiments showed that transfected MKL-1 overexpression vector, the mRNA level of cd44 after endogenous MKL-1 overexpression was significantly reduced. The above differences were statistically significant $(\mathrm{P}<0.05)$. In summary, the present study found that the expression and transcriptional activity of cd44 is dependent on MKL-1. However, the epigenetic mechanism governing the transcription of cd44 by MKL-1 remains to be further studied.

\section{References}

1. M. Alhajj, M.S. Wicha, A. Benitohernandez, S.J. Morrison, and M.F. Clarke.Proceedings of the National Academy of Sciences of the United States of America, 100, 3983-3988 (2003)

2. M. Jijiwa, H. Demir, S. Gupta, C. Leung, K. Joshi, N. Orozco, T. Huang, V.O. Yildiz, I. Shibahara, and J.A.D. Jesus.Plos One, 6, e24217 (2011)

3. S.I. Sayed, R.C. Dwivedi, R. Katna, A. Garg, K.A. Pathak, C.M. Nutting, P. Rhysevans, K.J. Harrington, and R. Kazi.Oral Oncology, 47, 237 (2011)

4. N. Sasaki, T. Ishii, R. Kamimura, M. Kajiwara, T. Machimoto, N. Nakatsuji, H. Suemori, I. Ikai, K. Yasuchika, and S. Uemoto.Cancer Letters, 308, 152161 (2012)

5. E.V. Abel, and A.E. Aplin.Frontiers in Bioscience, 3, 937-945 (2011)

6. L.S. Hart, N.G. Dolloff, D.T. Dicker, C. Koumenis, J.G. Christensen, A. Grimberg, and W.S. Eldeiry.Cell Cycle, 10, 2331-2338 (2011)

7. O. Naoki, and W.X. Wei.International Journal of Biological Sciences, 7, 517-535 (2011)
8. O. Nagano, and H. Saya.Cancer Science, 95, 930935 (2005)

9. C. Cheng, and P.A. Sharp.Molecular \& Cellular Biology, 26, 362 (2006)

10. V. D, V. M, K. E, R. M, M. P, G. A, C. M, H. VC, D.L. G, and P. A.Journal of Biological Chemistry, 283, 4448-4458 (2008)

11. L. Du, H. Wang, L. He, J. Zhang, B. Ni, X. Wang, H. Jin, N. Cahuzac, M. Mehrpour, and Y. Lu.Clinical Cancer Research, 14, 6751-6760 (2008)

12. S. Takaishi, T. Okumura, S. Tu, S.S.W. Wang, W. Shibata, R. Vigneshwaran, S.A.K. Gordon, Y. Shimada, and T.C. Wang.Stem Cells, 27, 1006-1020 (2009)

13. C. Zhang, C. Li, F. He, Y. Cai, and H. Yang.Journal of Cancer Research \& Clinical Oncology, 137, 1679 (2011)

14. F. Fang, Y. Yang, Z. Yuan, Y. Gao, J. Zhou, Q. Chen, and Y. Xu.Circulation Research, 108, 797807 (2011)

15. J. Rodriguez-Vita, M. Ruiz-Ortega, M. Rupã@Rez, V. Esteban, E. Sanchez-Lã ${ }^{3}$ Pez, J.J. Plaza, and J. Egido.Circulation Research, 97, 125-134 (2005)

16. N. Ramanan, Y. Shen, S. Sarsfield, T. Lemberger, G. Schütz, D.J. Linden, and D.D. Ginty.Nature Neuroscience, 8, 759-767 (2005)

17. F. Miralles, G. Posern, A.I. Zaromytidou, and R. Treisman.Cell, 113, 329-342 (2003)

18. C.R. Evelyn, E.M. Lisabeth, S.M. Wade, A.J. Haak, C.N. Johnson, E.R. Lawlor, and R.R. Neubig.Microarrays, 5, 13 (2016)

19. C. Hermanns, V. Hampl, K. Holzer, A. Aigner, J. Penkava, N. Frank, D.E. Martin, K.C. Maier, N. Waldburger, and S. Roessler.Oncogene, (2017)

20. P. Dalerba, S.J. Dylla, I.K. Park, R. Liu, X. Wang, R.W. Cho, T. Hoey, A. Gurney, E.H. Huang, and D.M. Simeone.Proceedings of the National Academy of Sciences of the United States of America, 104, 10158-10163 (2007)

21. C. Ginestier, H.H. Min, E. Charafejauffret, F. Monville, J. Dutcher, M. Brown, J. Jacquemier, P. Viens, C.G. Kleer, and S. Liu.Cell Stem Cell, 1, 555-567 (2007)

22. M. Kojima, G. Ishii, N. Atsumi, S. Fujii, N. Saito, and A. Ochiai.Cancer Science, 99, 1578-1583 (2008)

23. I. Gurbuz, J. Ferralli, T. Roloff, R. ChiquetEhrismann, and M.B. Asparuhova.Molecular Cancer, 13, 22 (2014)

24. X. Cheng, Y. Yang, Z. Fan, L. Yu, H. Bai, B. Zhou, $\mathrm{X}$. Wu, H. Xu, M. Fang, and A. Shen.Oncogene, 34, 5570 (2015)

25. H. Lei, D. Wu, J.Y. Wang, L. Li, C.L. Zhang, H. Feng, F.Y. Fu, and L.L. Wu.Basic Research in Cardiology, 110, 35 (2015)

26. M. Ishikawa, N. Nishijima, J. Shiota, H. Sakagami, K. Tsuchida, M. Mizukoshi, M. Fukuchi, M. Tsuda, 
and A. Tabuchi.Journal of Biological Chemistry, 285, 32734 (2010)

27. Z. Fan, C. Hao, M. Li, X. Dai, H. Qin, J. Li, H. Xu, $\mathrm{X}$. $\mathrm{Wu}, \mathrm{L}$. Zhang, and M. Fang.Biochimica et Biophysica Acta (BBA) - Gene Regulatory Mechanisms, 1849, 1219-1228 (2015) 